\title{
Comparison of cryoprotective methods for histological examination of rat and porcine lung tissue
}

\author{
Rea Jarošová1, Petra Ondráčková ${ }^{2}, Z_{\text {deněk Patočka }}^{3}, Z$ byšek Sládek ${ }^{1}$ \\ ${ }^{1}$ Mendel University in Brno, Faculty of AgriSciences, \\ Department of Morphology, Physiology and Animal Genetics, Brno, Czech Republic \\ ${ }^{2}$ Veterinary Research Institute, Department of Immunology, Brno, Czech Republic \\ ${ }^{3}$ Mendel University in Brno, Faculty of Forestry and Wood Technology, \\ Department of Forest Management and Applied Geoinformatics, Brno, Czech Republic
}

Received August 13, 2020

Accepted May 26, 2021

\begin{abstract}
Proper histological evaluation of lung tissue and accurate antigen detection by immunodetection techniques require histological tissue processing to preserve tissue reactivity and open alveolar spaces. In this study, we focused on testing and comparing different procedures of tissue cryopreservation. Sucrose or Tissue Tek were used with several methods of freezing samples by supercooled liquids and liquid nitrogen. Changes in tissue caused during the freezing of samples and the effect of cryoprotectants on the tissue were recorded. Rat and porcine pulmonary tissues were used in this experiment. This study aimed to optimize the process of lung cryopreservation with emphasis on enabling proper anatomical evaluation and preserving a high tissue immunoreactivity. The best results were obtained by inflating pulmonary parenchyma with a $1: 1$ mixture of O.C.T. with phosphate buffered saline (PBS) frozen in supercooled n-heptane placed on dry ice. Pulmonary tissue prepared in this way enables to perform proper histological evaluation and to detect target molecules by immunohistochemical analysis.
\end{abstract}

Alveolar microstucture, cryopreservation, frozen sections, immunohistochemistry, pig

Pulmonary tissue shows a very fragile and unique structure, formed by open alveolar spaces, where air exchanges between the organism and its environment. Under physiological conditions, lungs are filled with air that maintains a typical lung morphology. Lungs removed at necropsy normally collapse due to the loss of negative pleural pressure leading to a quite unnatural appearance of both gross and histological specimens (Hausmann et al. 2004). A proper histological evaluation of lung tissue is easier to perform if the alveolar spaces of the lungs are open. The deflated pulmonary parenchyma has a wider alveolar tissue structure resulting from inflammatory infiltrate. This is because the alveoli tend to collapse, which may appear as the presence of fibrosis or inflammation, thus providing a distorted picture for the pathological examination of the lungs (Churg 1983).

The method of formalin-fixed paraffin embedded tissue (FFPE) histology provides a good preservation of lung microstructure. Perfusion of lungs with buffered formalin at necropsy is an accepted technique to improve the histology of the pulmonary architecture (Hausman et al. 2004). On the other hand, the use of formalin reduces the immunoreactivity of many antigenic structures (Gatter et al. 1984). The process of processing paraffin slides also harms the quality of nucleic acid isolation, where formalin crosslinks between proteins and nucleic acids occur (Werner et al. 2000). Long-term storage of paraffin preparations causes considerable degradation of DNA, RNA, and many antigens (Von Ahlfen et al. 2007). A gentler and faster method of histological preparation is the frozen sections method (FS), which allows to leave out denaturation fixatives and keeps most of the biologically active substances in the native form, even in the long-term storage of samples. The FS provides

Address for correspondence:

Department of Morphology, Physiology and Animal Genetics

Faculty of AgriSciences, Mendel University in Brno

Zemědělská 1665/1, 61300 Brno, Czech Republic
E-mail: rea.jarosova@mendelu.cz

Phone: +420 545133152

http://actavet.vfu.cz/ 
ideal conditions for both immunological and molecular analyses, such as immunodetection or gene expression measurements (Gianoulis et al. 1988).

Preparing the tissue correctly is a basic step in the detection of antigenic molecules by using the immunohistochemical analysis (IHC), which serves to accurately locate, for example, inflammatory markers of immune cells during the study of respiratory diseases of pigs. At this time, porcine respiratory diseases, such as porcine respiratory and reproductive syndrome (PPRS) or pneumonia caused by actinobacillus pleuropneumoniae (APP), are currently a topic of animal research. Both of these diseases often cause great economic and breeding losses in swine breeding (Zimmerman et al. 2002). Identification of inflammatory processes in the lungs of infected animals, as well as an understanding of the interaction of the immune system with pathogens, can help to elucidate the pathogenesis and to improve protection of pigs against these diseases.

There are several established methods for the cryopreservative processing of lung tissue, and the selection of a suitable method depends on the target evaluation of preparation. After tissue sampling, specimens were portioned, and each portion handled and processed differently, to stabilize or preserve different biomolecules. Each specialized method preserves one class of biomolecules at the expense of others (Baatz et al. 2014).

Cryopreservation of the tissue is an important point in its treatment, as to avoid the formation of ice crystals arising when water, contained in the tissues, expands during solidification. Ice crystals disrupt cell membranes and cause so-called Swiss Cheese artifacts (Peters 2010). One way to prevent these problems is to treat the tissue with a suitable cryoprotectant which interferes with interactions between the polar bonds of the water molecule. Most commonly used cryoprotectants are sucrose, glycerol, and polyethylene glycol (Pegg 2007). Sucrose was used for tissue cryopreservation by many authors (Vacciana et al. 1994) in combination with formaldehyde (Hosoda et al. 2001) or 4\% paraformaldehyde (Ling et al. 2009).

For proper evaluation of lung tissue it is necessary to maintain open alveolar spaces using the instillation of cryoprotective media into inner spaces of the lung. There are many different preservative options for filling lung tissue with various cryoprotectants (Baijnath et al. 2016). A former study (Rabinovitch et al. 1981) reports the filling of the pulmonary parenchyma with glutaraldehyde. In a another study (Halbower et al. 1994), preservation of alveolar structure was performed by filling lungs with agarose. In a different study (Braber et al. 2010), formalin together with agarose was used for fixation of the lung microstructure in mice.

Pulmonary parenchyma cryopreservation by using sucrose and commercially produced cryoprotective polymer Tissue Tek "optimal cutting temperature" compound (O.C.T. Compound; Sakura-Finetek, Tokyo, Japan) was tested. Moreover, some methods of tissue freezing in supercooled liquids (isopentane, n-heptane), in liquid nitrogen or freezing in a cryostat were sampled and compared. The aim of this study was the preservation of alveolar microstructure of lungs and maintaining high tissue immunoreactivity. Anticipated results were to minimize the compromise between selection of molecular or protein analysis and anatomical evaluation, as well as to provide a simple and reproducible method of tissue preparation.

\section{Materials and Methods}

Experimental material

The experiment was performed in compliance with the law of the Czech Republic (Act No. 501/2020 Coll.) for the protection of animals against cruelty and with the agreement of the Branch Commission for Animal Welfare of the Ministry of Agriculture of the Czech Republic (approval no. 31674/2018-MZE-17214).

Eight lungs of laboratory rats (Rattus norvegicus) were used for this experiment. Rats were anaesthetized with ether and sacrificed by cranio-cervical dislocation. After dissection, isolation of whole lungs was performed, the pulmonary tissue was portioned and processed by several different cryopreservation procedures. A standard 
fixation of the samples by $10 \%$ formalin was omitted in order to preserve lipids and proteins in their native form.

Additionally, the lungs of two eight-week-old pigs (Sus scrofa f. domestica) without anomaly or vaccination were used. The pigs were kept in the accredited barrier-type animal facilities of the Veterinary Research Institute (VRI). Before being sacrified, the pigs were allowed to acclimatize in animal facilities for one week. Experimental material was collected by trained staff from an authorized and registered slaughterhouse at VRI. Following the isolation of the whole lungs, pulmonary tissue treatment was performed immediately using optimized cryopreservative methods which were found in the rat models.

Experimental design

Sampling of rat pulmonary tissues

Cryopreservation by a $10 \%$ sucrose (S) (Ha \& Ka CZ s.r.o., Mnichovo Hradiště, Czech Republic) solution were tested. Parts of the lung samples were immersed in a $10 \% \mathrm{~S}$ solution at $4{ }^{\circ} \mathrm{C}$ for $24 \mathrm{~h}$ overnight $(\mathrm{O} / \mathrm{N})$. Other lung samples were inserted into O.C.T and frozen. For enabling the preservation of open alveolar spaces, some lung samples were filled with $10 \% \mathrm{~S}$ solution, others by O.C.T. The cryoprotective materials were injected into airways by cannulation of the bronchi. Lungs samples were cut and tissue blocks were trimmed to the size of $5 \times 5 \times 5 \mathrm{~mm}$ and then frozen.

The freezing of samples was performed in four different ways. Samples were frozen in liquid nitrogen (LN), in supercooled isopentane (n-pentane) (n-P) (PENTA s.r.o., Praha, Czech Republic), and n-heptane (n-H) (PENTA s.r.o.) placed on dry ice or frozen in the cryomicrotome $(\mathrm{CM})$ at $-20{ }^{\circ} \mathrm{C}$. Metal containers with a capacity of $250 \mathrm{ml}$ were used for freezing in supercooled liquids. One of the containers was filled with $200 \mathrm{ml}$-P, the second with $200 \mathrm{ml} \mathrm{n}-\mathrm{H}$. These metal containers were wholly embedded into dry ice and liquids were pre-cooled for $30 \mathrm{~min}$. Samples were embedded into the vessel of an aluminum foil and some of them contained O.C.T. All samples were frozen. During freezing, samples were held beneath the surface of the liquid.

Two tissue blocks were used from each cryopreservation procedure. Samples frozen in the CM were stored at $-20{ }^{\circ} \mathrm{C}$. Samples frozen in LN and super-cooled liquids (n-P and $\left.\mathrm{n}-\mathrm{H}\right)$ were stored at $-80{ }^{\circ} \mathrm{C}$. Ten various combinations of tissue cryopreservation were tested in this part of the experiment.

Variants of rat pulmonary tissue processing

Ra1 samples were frozen in supercooled n-H without any cryoprotective treatment.

Ra2 samples were immersed in a $10 \% \mathrm{~S}$ solution $\mathrm{O} / \mathrm{N}$ and frozen in supercooled $\mathrm{n}-\mathrm{H}$.

$\mathrm{Ra} 3$ samples were immersed in a $10 \% \mathrm{~S}$ solution $\mathrm{O} / \mathrm{N}$ and frozen in the $\mathrm{CM}$.

Ra4 lung inflation and imersion by a $10 \% \mathrm{~S}$ solution $\mathrm{O} / \mathrm{N}$ and frozen in supercooled $\mathrm{n}-\mathrm{H}$.

Ra5 lung inflation and imersion by a $10 \% \mathrm{~S}$ solution $\mathrm{O} / \mathrm{N}$ and frozen in the $\mathrm{CM}$.

Ra6 samples were embedded in O.C.T. and frozen in LN.

Ra7 samples were embedded in O.C.T. and frozen in supercooled n-H.

Ra8 lung inflation with O.C.T., embedded in O.C.T. and frozen in LN.

Ra9 lung inflation with O.C.T., embedded in O.C.T. and frozen in supercooled n-P.

Ra10 lung inflation with O.C.T., embedded in O.C.T. and frozen in supercooled n-H.

\section{Sampling of porcine pulmonary tissues}

Porcine lung tissue was processed by only those procedures that showed the best results in rats. For cryopreservation, only O.C.T was used; the freezing of the samples was done in LN and n-H. Inflation of lungs with O.C.T. was optimized by diluting O.C.T. in phosphate buffered saline (PBS) (Bio Whittaker, Lonza, Basel, Switzerland) at a ratio of 1:1. The process of freezing and storage of porcine samples was performed in the same way as mentioned above.

\section{Processing of porcine lungs}

After lung isolation, caudal right and left lung lobes were selected. The first step was the trimming of the lung lobe into large blocks of approximately 5-10 $55-10 \times 5-10 \mathrm{~cm}$, then cryoprotective medium was instilled into bronchi, and the tissue was cut into blocks and trimed to the size of 5-7 $\times 5-7 \times 5-7 \mathrm{~mm}$ before being frozen. Three tissue blocks from each procedure were used in this part of the experiment.

Variants of porcine pulmonary tissues processing

Su1 samples were embedded in O.C.T. and frozen in LN.

$\mathrm{Su} 2$ samples were embedded in O.C.T. and frozen in supercooled n-H.

Su3 lung inflation with pure O.C.T., embedded in O.C.T. and frozen in supercooled n-H.

Su4 lung inflation by diluted O.C.T., embedded in O.C.T. and frozen in supercooled n-H.

Su5 lung inflation by diluted O.C.T, embedded in O.C.T. and frozen in LN.

Cryohistology

The tissue samples were cut to a thickness of 5-10 $\mu \mathrm{m}$ on the cryostat (Leica Microsystems, CM 1900, GmbH, Wetzlar, Germany) at a temperature of $-20{ }^{\circ} \mathrm{C}$. The cuts of tissue were placed on slides, the sections allowed to dry at room temperature, fixed in pre-cooled acetone (PENTA s.r.o.) at $-18^{\circ} \mathrm{C}$ for 5 min and stained with Mayer 
haematoxylin (MH) (PENTA s.r.o.). A total of 140 histological slides of 4 sections each were evaluated using the light microscope Olympus BH-2 (Olympus, Tokyo, Japan) at $\times 100$ and $\times 400$ magnifications. Images of sections were taken with a VH-Z500R high resolution zoom lens (Keyence, Itasca, U.S.A.) mounted on the Keyence VHX-5000 digital microscope (Keyence). Two magnifications, $\times 500$ and $\times 1000$ were used.

Immunohistochemistry

Immunohistochemical analysis (IHC) confirms detection of antigens in the cryopreserved tissue. The endogenous peroxidase was blocked with Dual Endogenous Enzyme-Blocking Reagent (DAKO, Glostrup, Denmark) for $10 \mathrm{~min}$. The slides were washed and the Protein Block (DAKO) applied for 5 min. Then, anti-CD163 (Serotec, Oxford, UK), the antibody was applied and the slides were incubated for 60 min at $37^{\circ} \mathrm{C}$ in a humid chamber. The slides were washed and EnVision reagent (HRP, Mouse, DAKO) was added. The slides were incubated for $30 \mathrm{~min}$ at $37^{\circ} \mathrm{C}$ in a humid chamber. Then they were washed and stained with $\mathrm{DAB}+(\mathrm{Liquid}$, DAKO) for approximately $30 \mathrm{~s}$. The next step was washing the slides with distilled water, lightly counterstained with $\mathrm{MH}$ and mounted in glycerol-gelatine. As a negative control, immunohistochemistry was performed using secondary antibodies without the primary antibody. Preservation of the lung microstructure was the primary task. Subsequent IHC staining was performed only in samples treated by the procedure with the best cryopreservative properties and for a confirmation of tissue immunoreactivity.

\section{Results}

\section{Photomicrographic assessment of rat lung parenchyma}

In the Ral samples (frozen in supercooled n-H without any cryoprotective treatment), the alveolar structure was not preserved. Alveoli were totally collapsed, showing atelectasis (Plate XIX, Fig. 1A, detail 1a).

In Ra2 samples (immersed in a $10 \% \mathrm{~S}$ solution $\mathrm{O} / \mathrm{N}$ and frozen in supercooled $\mathrm{n}-\mathrm{H}$ ), a total destruction of the pulmonary parenchyma occurred. Haematoxylin precipitate was observed, complicating the histological evaluation of the sample (Fig. 1B, detail 1b).

In Ra3 samples (immersed in a $10 \% \mathrm{~S}$ solution $\mathrm{O} / \mathrm{N}$ and frozen in the $\mathrm{CM}$ at $-20{ }^{\circ} \mathrm{C}$ ), the alveolar structure was not preserved. Collapsed alveoli were observed, cells and cell nuclei were not distinguishable. Swiss cheese artifacts were found (Fig. 1C, detail 1c).

In Ra4 samples (lung inflation and immersion in a $10 \% \mathrm{~S}$ solution $\mathrm{O} / \mathrm{N}$ and frozen in supercooled $\mathrm{n}-\mathrm{H})$, the alveolar structure was not preserved. Collapsed alveoli were observed, cells and cell nuclei were not distinguishable. Swiss cheese artifact and air bubbles in cryoprotective medium were found (Fig. 1D, detail 1d).

In Ra5 samples (lung inflation and imersion in a $10 \% \mathrm{~S}$ solution $\mathrm{O} / \mathrm{N}$ and frozen in the $\mathrm{CM}$ at $-20^{\circ} \mathrm{C}$ ), a little partial contour of the alveolar structure was shown. Due to a very blurred microstructure, it was not possible to distinguish alveoli, intraalveolar septa or cell nuclei. Haematoxylin precipitate and air bubbles in cryoprotective medium were found (Fig. 1E, detail 1e).

In Ra6 samples (embedded in O.C.T. and frozen in LN), the alveolar structure was preserved. The microstructure was very blurred. Dense cellularity was observed in some areas. Some connective tissue was separated. Cell nuclei could not be observed in poorly visible cells (Plate XX, Fig. 2F, detail 2f).

In Ra7 samples (embedded in O.C.T. and frozen in supercooled n-H), poor quality of alveolar structure with blurred microstucture was observed. Alveolar walls and intraalveolar septa were wider, some alveoli were collapsed. Densely cellular areas were found. Pneumocytes were difficult to distinguish, macrophages were without visible nuclei. Haematoxylin precipitate was present (Fig. 2G, detail 2g).

In Ra8 samples (lung inflation with O.C.T., embedded in O.C.T. and frozen in LN), the alveolar structure was preserved. Unnaturally enlarged intraalveolar septa were observed. The connective tissue was separated from alveolar walls. Cells were difficult to distinguish. Contamination with unfamiliar particles was found (Fig. $2 \mathrm{H}$, detail $2 \mathrm{~h}$ ).

In Ra9 samples (lung inflation with O.C.T., embedded in O.C.T. and frozen in supercooled $\mathrm{n}-\mathrm{P})$, the alveolar structure was preserved. Collapsed alveoli were observed, cells and cell nuclei could not be distinguished properly. The connective tissue was separated from 
alveolar walls. Massive contamination with separated connective tissue and unfamiliar particles was found (Fig. 2I, detail 2i).

In Ra10 samples (lung inflation with O.C.T., embedded in O.C.T. and frozen by supercooled $\mathrm{n}-\mathrm{H}$ ) the alveolar structure was preserved. Alveoli and intraalveolar septa had a natural appearance. Some connective tissue was separated from alveolar walls. Cells in alveolar walls could be distinguished (Fig. 2J, detail 2j).

\section{Photomicrographic assessment of porcine lung parenchyma}

In Sul samples (embedded in O.C.T. and frozen in LN), the alveolar structure was not preserved. Alveoli were collapsed, showing partial atelectasis. Massive dense cellularity was observed (Plate XXI, Fig. 3A, detail 3a).

In $\mathrm{Su} 2$ samples (embedded in O.C.T. and frozen in supercooled n-H) preserved alveolar structure was found. Some alveoli were collapsed. Cells and cell nuclei were visible and could be distinguished (Fig. 3B, detail 3b).

In Su3 samples (lung inflation with O.C.T., embedded in O.C.T. and frozen in supercooled $\mathrm{n}-\mathrm{H}$ ), preserved alveolar structure was found. Some collapsed alveoli and torn areas were observed. Visible cell and cell nuclei could be distinguished (Fig. 3C, detail 3c).

In $\mathrm{Su} 4$ samples (lung inflation by diluted O.C.T., embedded in O.C.T. and frozen in supercooled $n-H)$, preserved alveolar structure was found. A natural appearance of alveoli and an optimal thickness of alveolar walls were observed. Visible cell and cell nuclei could be distinguished (Fig. 3D, detail 3d).

In Su5 samples (lung inflation by diluted O.C.T, embedded in O.C.T. and frozen in $\mathrm{LN})$, the alveolar structure was preserved. Unnaturally enlarged intraalveolar septa were observed. Cells and cell nuclei were visible and could be distinguished (Fig. 3E, detail 3e).

The difference between the inflated part and collapsed part of the interalveolar septa, thickening by extensive inflammatory cell infiltration in rat and in porcine lungs is shown in Fig. 4A and B (Plate XXII).

\section{Immunohistochemistry}

IHC analysis was performed only in the best cryopreserved pulmonary tissue by the method of lung inflation by diluting O.C.T., embedding in O.C.T., and freezing in supercooled n-H. Open and well-visible alveolar spaces enabled accurate detection and space localization of the CD163 molecule in porcine lungs (Fig. 4C, D).

\section{Discussion}

This study aimed to compare methods of lung cryopreservation and furthermore, to find a simple and applicable procedure for tissue cryopreservation, and to create useful histological specimen that could be properly evaluated. Pulmonary rat and porcine tissues were used in this experiment. First, cryopreservation of the rat lung tissue was tested. The rat was selected for experimental purposes due to low cost and quick and easy acquisition of the biological material. The purpose of this experiment was to establish an adequate preservation method of the porcine lung parenchyma and subsequently, to determine the space localization of immunological markers.

The sections showed that the method of freezing samples without any cryoprotective treatment led to the atelectasis, collapsed airways, and also alveoli (Fig. 1A). This method was included for identifying and assessing changes that occurred during the freezing of untreated tissue, and for a subsequent comparison with cryopreserved tissues. According to the Bearer and Orci (1986), the tissue can be frozen without cryoprotection, but freezing must be done as quickly as possible with a vapour of liquid nitrogen. 
Cryopreservation by a $10 \%$ sucrose solution was tested. By using sucrose, we assumed that the tissue would be protected from the formation of ice crystals and the tissue tension would be maintained. The effect of $10 \%$ sucrose solution was totally destructive for the lung parenchyma (Fig. 1B-E) and did not prevent the formation of ice crystals (Fig. 1C, D). Inflation with a $10 \%$ sucrose solution did not preserve the alveolar microstructure (Fig. 1D, E). In contrast to Hosoda et al. (2001) who used $20 \%$ sucrose in combination with formalin in their study, we did not achieve satisfactory results. In our experiment, formalin fixation was omitted to preserve lipids and proteins in their native form for subsequent IHC analysis.

Lung samples cryopreserved only by insertion into O.C.T. and freezing showed a poor quality of the alveolar microstructure. The alveolar walls appeared to be much wider than in their physiological state (Fig. 2F, G, B) or showed totally collapsed alveoli (Fig. 3 A). We chose the method of inflation of bronchoalveolar spaces with pure embedding media O.C.T. according to Gianoulis et al. (1988) for the preservation of lung morphology. The alveolar microstructure was partially preserved during this procedure. The connective tissue was separated from the alveolar walls (Fig. $2 \mathrm{H}-\mathrm{J}$ ), some parts of the microstructure were torn (Fig. $3 \mathrm{C}$ ) probably due to the high density of the media. The density of O.C.T. was not suitable for inflation, especially in the rat lungs which are smaller and less accessible than the porcine lungs. Similarly to Myung et al. (2008), we tested the dilution of O.C.T. with PBS at a ratio of 1:1. In comparison with Prince and Porter (1975) who used a 1:2 mixture of O.C.T. and PBS injected intratracheally into fresh lung tissue, our histological sections showed that a 1:1 dilution of O.C.T. with PBS was sufficient. The specimens showed correctly preserved alveolar microstructure. Clear and sharp alveolar walls had optimal thickness, well-visible nuclei in cells, and a minimum of freezing artifacts (Fig. 3D, E). In this case, the smallest negative impact on the lung parenchyma microstructure was found. This embedding medium inflation technique may be applied to basic research. Morphology preserved in the frozen tissue may be helpful in pertinent interpretation of molecular data.

Several methods of freezing samples were tested in this experiment. Sections showed that freezing in the cryomicrotome at $-20{ }^{\circ} \mathrm{C}$ produced artifacts in the form of white holes, the so called Swiss Cheese artifacts (Fig. 1C). Similarly Peters (2003) reported that the slow freezing of tissue samples in the cryostat caused numerous artifacts. Swiss Cheese artifacts appeared also in a speciment frozen in n-heptane (Fig. 1D), probably due to improperly pre-cooled freezing media and by the slow freezing process.

Better results were obtained by freezing samples in supercooled liquids isopentane and $\mathrm{n}$-heptane placed on dry ice. Both procedures provided uniform freezing, adequate freezing rate, and no cracks in frozen samples. Due to numerous contaminations of the samples frozen in n-pentane (Fig. 2I), we selected only n-heptane for subsequent freezing. Similar results were obtained by Peters (2003). Faster freezing of the sample in liquid nitrogen or super-cooled liquids limits the creation of frosty artifacts but also limits the precision of isolation. Our results showed that freezing in liquid nitrogen was an aggressive method for the pulmonary tissue. The samples often cracked during the freezing and the evaluated sections had a widespread alveolar structure probably due to the boiling of the liquid nitrogen (Fig. 2H, 3E).

Some sections revealed the interalveolar septum inflated by diluted O.C.T. with a preserved alveolar microstucture in contrast to the adjacent collapsed part of the septum, where collapsed alveoli resulting in dense cellularization (Fig. 4A, B).

Pulmonary tissue processed by the inflation method with diluted O.C.T. and frozen by supercooled n-heptane showed the most suitable parameters for IHC analysis (Fig. 4C, D). In this part of the experiment, antigen CD163 was chosen as a membrane sign of myeloid cells such as monocytes/macrophages that naturally occurred in lungs. Antigen CD163 was 
used to detect alveolar mononuclear phagocytes and confirmed the preservation of tissue immunoreactivity.

In summary, we have described a simple technique for lung tissue inflation by embedding medium O.C.T. diluted with PBS at a ratio of 1:1 and freezing in supercooled n-heptane placed on dry ice. This method is simple and quick to perform in comparison with other methods, providing perceptible morphological details of lung microstructure, and enabling alveoli to be held open, thus making a thorough pathological evaluation possible, with a quality comparable to paraffin sections. Tissue prepared in this way allows the detection and localization of the antigen by using the immunohistochemistry method. Optimizing cryopreservation of the pulmonary tissue will lead to a better detection of pathological processes in lungs when studying respiratory diseases of pigs.

\section{Conflict of interest}

The authors declare no conflict of interest.

\section{Acknowledgements}

This research project AF-IGA-IP-2018/021 was financially supported by the Internal Grant Agency of Mendel University in Brno and the Ministry of Agriculture of the Czech Republic (RO 0519).

\section{References}

Baatz JE, Newton DA, Riemer EC, Denlinger CE, Jones EE, Drake RR, Spyropoulos DD 2014: Cryopreservation of viable human lung tissue for versatile post-thaw analyses and culture. In Vivo 28: 411-423

Baijnath S, Shobo A, Bester LA, Singh SD, Kruger G, Naicker T, Govende T 2016: Small molecule distribution in rat lung: a comparison of various cryoprotectants as inflation media and their applicability to MSI. J Mol Histol 47: 213

Bearer EL, Orci L 1986: A simple method for quick-freezing. J Electron Microsc Tech 3: 233-241

Braber S,Verheijden KAT, Henricks PAJ, Kraneveld AD, Folkerts G 2010: A comparison of fixation methods on lung morphology in a murine model of emphysema. Am J Physiol Lung Cell Mol Physiol 299: L843-L851

Churg AMD 1983: An inflation procedure for open lung biopsies. Am J Surg Pathol 7: 69-72

Gatter KC, Falini B, Mason DY 1984: The use of monoclonal antibodies in histopathological diagnosis. Recent advances in histopathology. London, Churchill Livingstone, pp. 35-68

Gianoulis M, Chan N, Wright JL 1988: Inflation of lung biopsies for frozen section. Mod Pathol 1: 357-358

Halbower AC, Mason RJ, Abman SH, Tuder RM 1994: Agarose infiltration improves morphology of cryostat sections of lungs. Lab Invest 71: 149-153

Hausmann R, Bock H, Biermann T, Betz P 2004: Influence of lung fixation technique on the state of alveolar expansion - a histomorphometrical study. Leg Med (Tokyo) 6: 61-65

Hosoda H, Shinohara N, Kojima N, Maruyama K, Handa Y, Uchida M, Koike H, Kobayashi Y 2001: Contrivance for frozen section diagnosis of early minute lung cancer during operation. Kyobu Geka 54: 913-916

Ling LH, Rogers SM, Tay V, Limmon GV, Dahai Z, Roger K 2009: Comparison of various tissue-preparation techniques for cryosectioning of frozen section mouse tissues. J Histotechnol 32: 186-189

Myung JK, Choe G, Chung DH, Seo JW, Jheon S, Lee CT, Chung JH 2008: A simple inflation method for frozen section diagnosis of minute precancerous lesions of the lung. Lung Cancer 59: 198-202

Pegg DE 2007: Principles of cryopreservation. Methods Mol Biol 368: 39-57

Peters SR 2003: The art of embedding tissue for frozen section. Part I: A system for face down cryoembedding of tissues using freezing temperature embedding wells. J Histotechnol 26: 11-19

Peters SR 2010: A practical guide to frozen section technique. Springer, New York, pp. 97-115

Prince AG, Porter DD 1975: Cryostat microtomy of lung tissue in an expanded state. Stain Technology 50: 43-45

Rabinovitch M, Castaneda AR, Reid L 1981: Lung biopsy with frozen section as a diagnostic aid in patients with congenital heart defects. Am J Cardiol 47: 77-84

Vacciana A, Ebeigbe AB, Hasse J, Swoboda L, Müller-Schweinitzer E 1994: Evidence for preservation of epithelial function in cryopreserved porcine and human bronchi. Exp Physiol 79: 409-414

Von Ahlfen S, Missel A, Bendrat K, Schlumpberger M 2007: Determinants of RNA quality from FFPE samples. PLoS One 5;2: e1261

Werner M, Chott A, Fabiano A, Battifora H 2000: Effect of formalin tissue fixation and processing on immunohistochemistry. Am J Surg Pathol 24: 1016-1019

Zimmerman JJ, Karriker LA, Ramirez A, Schwartz KJ, Stevenson GW 2002: Diseases of Swine. $10^{\text {th }}$ edn, Iowa: Wiley, Ames, 685, 749 p. 
Plate XIX

Jarošová R. et al.: Comparison ... pp. 225-231
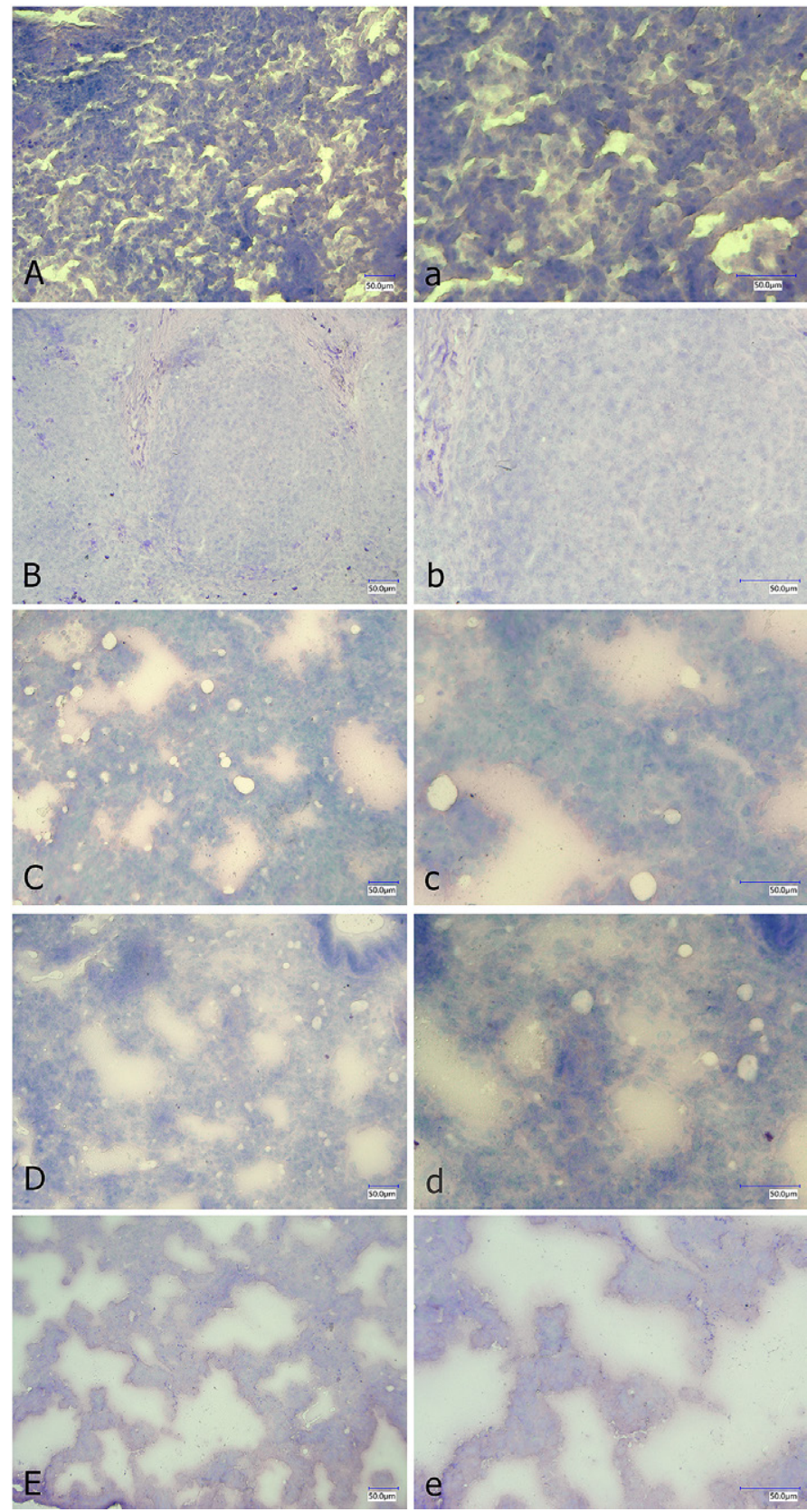

Fig. 1. Mayer haematoxylin stained frozen section of rat lungs: A - frozen by supercooled n-heptane (n-H) without any treatment by cryo-protective agent, detail (a); B - lungs immersed in a solution of $10 \%$ sucrose (S) O/N and frozen by supercooled $\mathrm{n}-\mathrm{H}$, detail (b); $\mathrm{C}$ - immersed in a $10 \% \mathrm{~S}$ at $\mathrm{O} / \mathrm{N}$ and frozen in cryomicrotome $(\mathrm{CM})$ at $-20{ }^{\circ} \mathrm{C}$, detail (c); D - instillation of a $10 \% \mathrm{~S}$, immersed $\mathrm{O} / \mathrm{N}$ and frozen by supercooled $\mathrm{n}-\mathrm{H}$, detail (d); $\mathrm{E}$ - instillation of $10 \% \mathrm{~S}$, immersed $\mathrm{O} / \mathrm{N}$ and frozen in the $\mathrm{CM}$ at $-20{ }^{\circ} \mathrm{C}$, detail (e). 


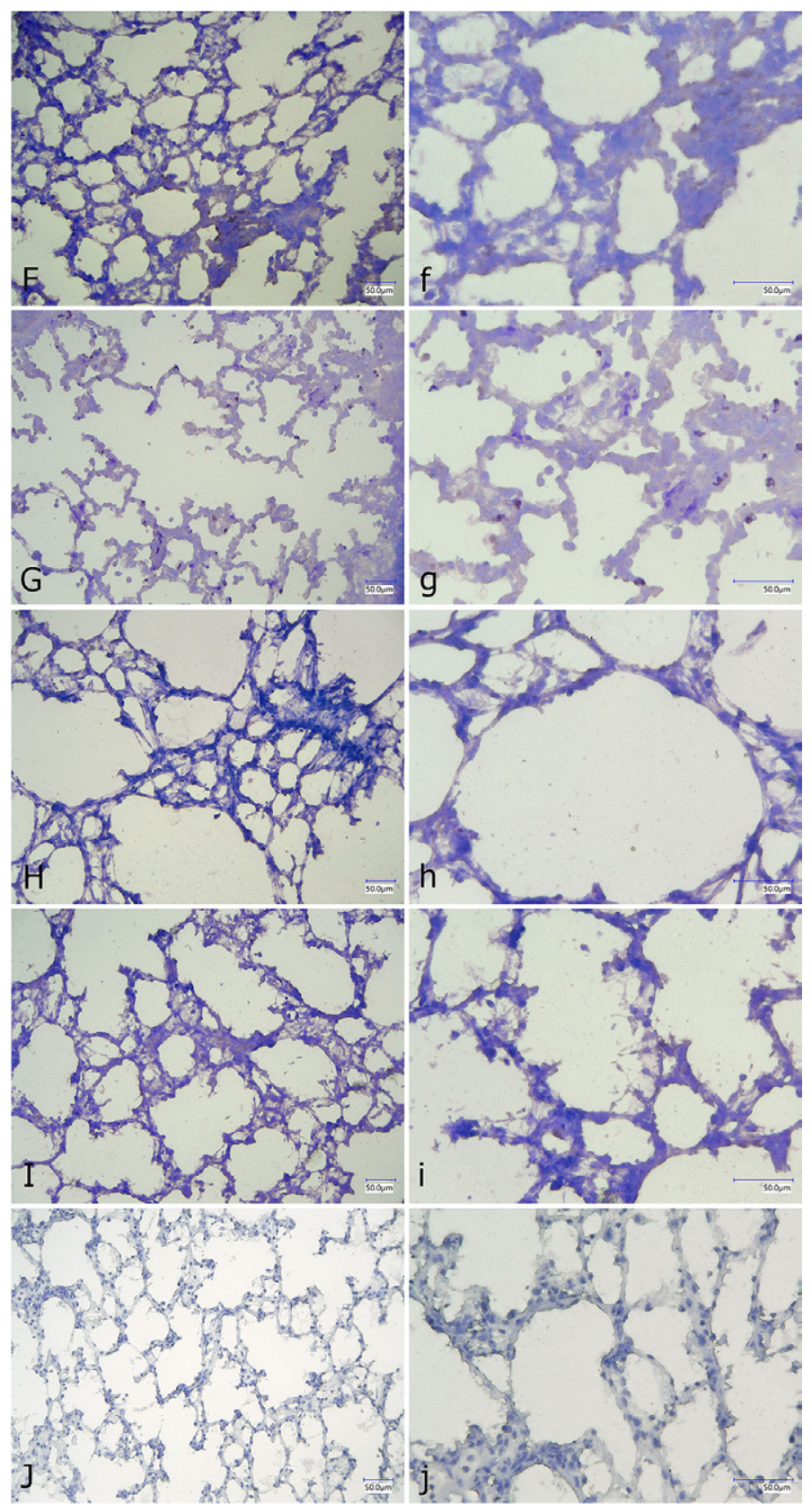

Fig. 2. Mayer haematoxylin stained frozen section of rat lungs: F - embedded in optimal cutting temperature compound (O.C.T.) and frozen in liquid nitrogen (LN), detail (f); G - embedded in O.C.T. and frozen by supercooled n-heptane (n-H), detail (g); H - instillation of O.C.T. and frozen in LN, detail (h); I - instillation of O.C.T. and frozen by supercooled n-pentane (n-P), detail (i); J - instillation of O.C.T. and frozen by supercooled $\mathrm{n}-\mathrm{H}$, detail (j). 


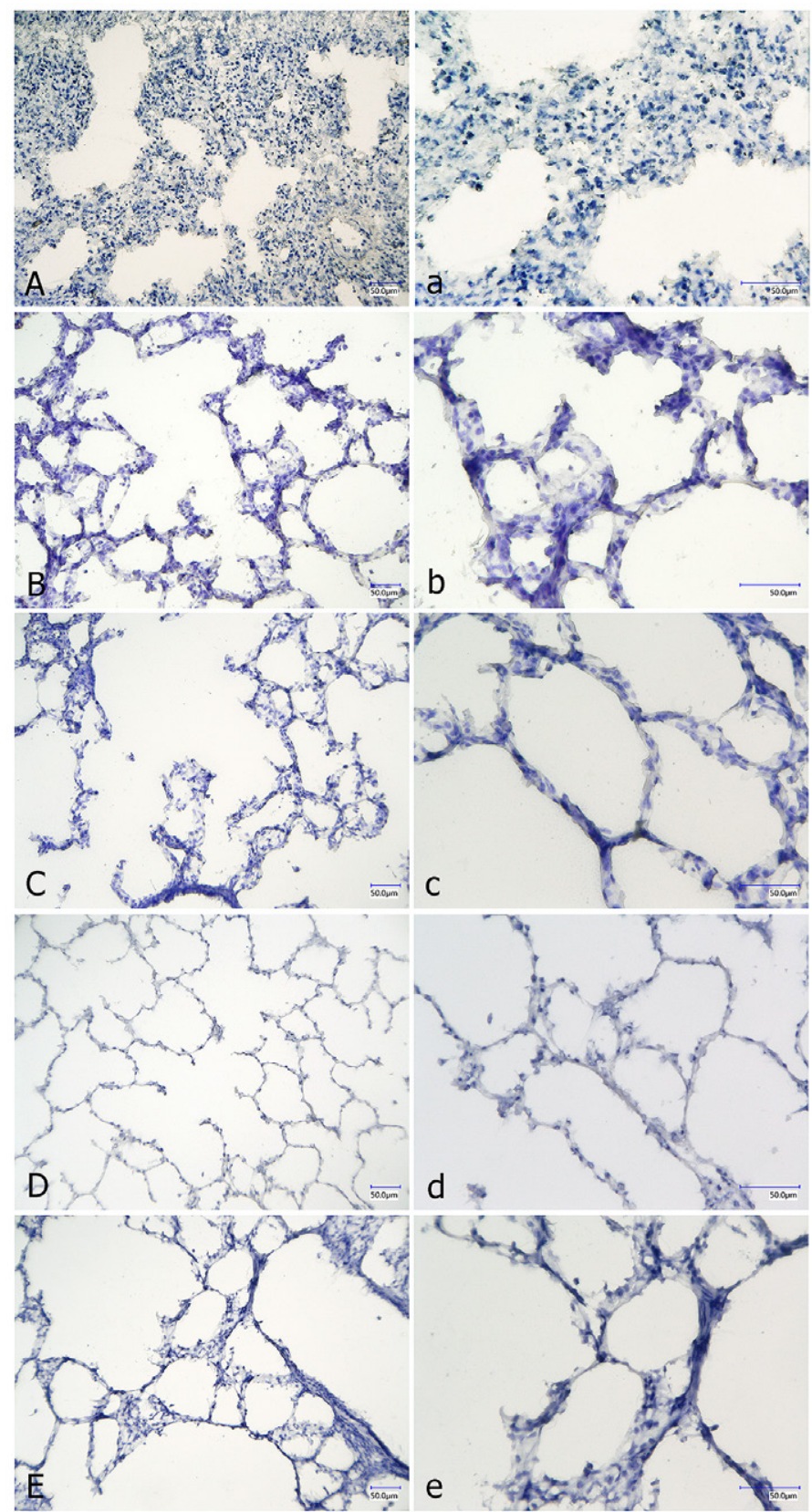

Fig. 3. Mayer haematoxylin stained frozen section of porcine lungs: A - embedded in optimal cutting temperature compound (O.C.T.) and frozen in liquid nitrogen (LN), detail (a); B - embedded in O.C.T. and frozen by supercooled n-heptane (n-H), detail (b); C - instillation of O.C.T., embedded in O.C.T. and frozen by supercooled $\mathrm{n}-\mathrm{H}$, detail (c); D - instilaltion of diluted O.C.T., embedded in O.C.T. and frozen in supercooled n-H, detail (d); E - instilaltion of diluted O.C.T., embedded in O.C.T. and frozen LN, detail (e). 


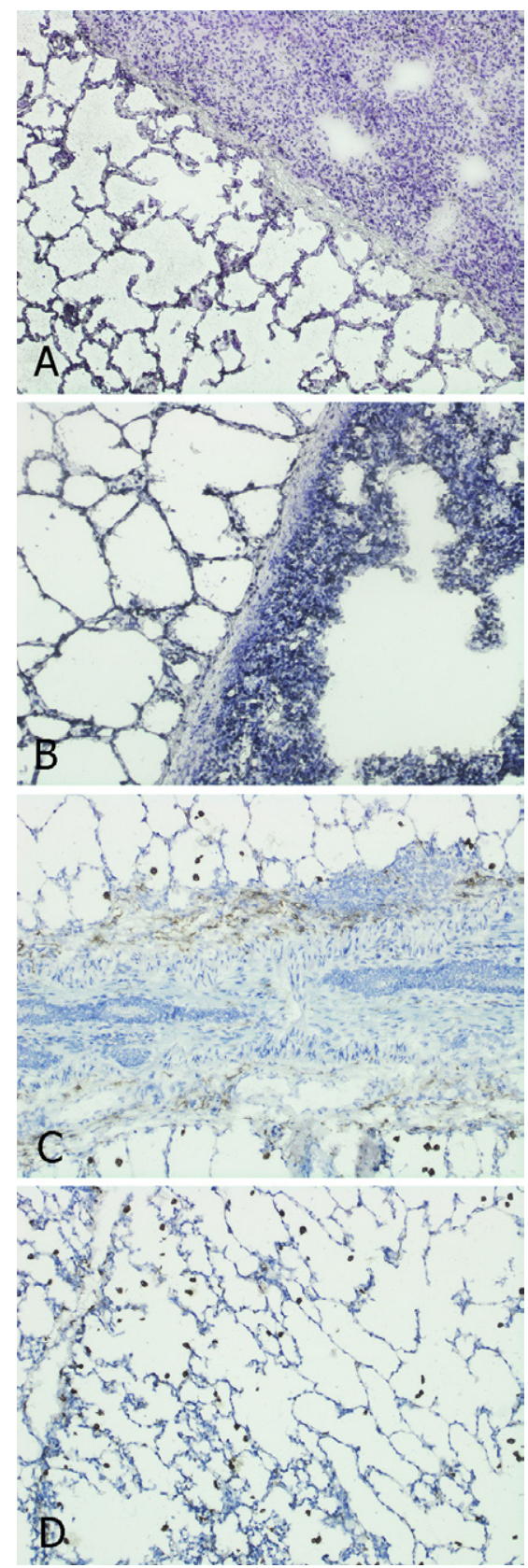

Fig. 4. A - inflated interalveolar septum shows preserved alveolar microstucture in contrast to adjacent collapsed part of septum, with collapsed alveoli resulting in dense cellularization in rat lung, (B) in porcine lung. Magnification $\times 100$. Immunohistochemical localization of the CD163 molecule on frozen section of porcine lungs inflated with a mixture of optimal cutting temperature compound (O.C.T.) and phosphate buffered saline (PBS) (1:1). C - interalveolar septum and connective tissue. D - alveoli. CD163 positive cells are stained by brown colour. Magnification $\times 100$. 